\title{
Meiotic And Early Zygotic Development In Crassostrea Virginica Observed Through Confocal Microscopy
}

H Masaru

A Komaru

Joana Teixeira De Sousa

Virginia Institute of Marine Science

Standish K. Allen Jr.

Virginia Institute of Marine Science

Follow this and additional works at: https://scholarworks.wm.edu/vimsarticles

Part of the Marine Biology Commons

\section{Recommended Citation}

Masaru, H; Komaru, A; De Sousa, Joana Teixeira; and Allen, Standish K. Jr., "Meiotic And Early Zygotic Development In Crassostrea Virginica Observed Through Confocal Microscopy" (2017). VIMS Articles. 301.

https://scholarworks.wm.edu/vimsarticles/301

This Article is brought to you for free and open access by the Virginia Institute of Marine Science at W\&M ScholarWorks. It has been accepted for inclusion in VIMS Articles by an authorized administrator of W\&M ScholarWorks. For more information, please contact scholarworks@wm.edu. 


\title{
MEIOTIC AND EARLY ZYGOTIC DEVELOPMENT IN CRASSOSTREA VIRGINICA OBSERVED THROUGH CONFOCAL MICROSCOPY
}

\author{
HOTTA MASARU, ${ }^{1}$ AKIRA KOMARU, ${ }^{1}$ JOANA TEIXEIRA DE SOUSA ${ }^{2}$ \\ AND STANDISH K. ALLEN JR. ${ }^{2 *}$ \\ ${ }^{1}$ Faculty of Bioresources, Mie University, 1577 Kurimamachiya-cho, Tsu-city, Mie, 514-8507, Japan; \\ ${ }^{2}$ Aquaculture Genetics and Breeding Technology Center, Virginia Institute of Marine Science, College of \\ William and Mary, 1375 Greate Road, Gloucester Point, VA 23062
}

\begin{abstract}
Several studies have examined early development in oysters, but few have reported the normal (expected) behavior of meiosis and early mitosis or of the organization of the spindle by the centrosomes. To establish this baseline, normal development of fertilized eggs in Crassostrea virginica from meiosis until the four-cell stage using confocal microscopy was documented. To visualize the centrosomes and microtubules, eggs were stained with antibodies for $\gamma$ - and $\alpha$-tubulin. In addition to establishing normal development, two additional features of early development were documented. First, centrosome behavior was documented. The centrosome associated with the female pronucleus disappeared after polar body formation. The single centrosome associated with the male pronucleus duplicated by late anaphase of meiosis II to eventually form the spindle for mitosis I, thus the two centrosomes in the first cleavage were derived from the sperm. Second, linkage between the cell and centrosome cycles was established. In the first and second mitosis, centrosome duplication began at early anaphase. At late anaphase, the centrosomes duplicated and were observed as two distinct units at each pole. Asynchrony was also observed at the four-cell stage between the large and small blastomeres, with chromatin and centrosome duplication showing more advanced stages in the large blastomere. This documentation will serve as a baseline for future studies on chromosome loss in polyploid oysters.
\end{abstract}

KEY WORDS: oyster, Crassostrea virginica, centrosome, microtubule, meiosis, cleavage

\section{INTRODUCTION}

Chromosome loss in Crassostrea species had been documented for both diploids (Thiriot-Quiévreux et al. 1992, Leitão et al. 2001, de Sousa et al. 2011) and polyploids (Allen et al. 1996, McCombie et al. 2005, Zhang et al. 2010, Zhang et al. 2014). In a previous study on the subject, de Sousa et al. (2016) hypothesized the possible involvement of supernumerary centrosomes in early development and possibly in later ontogeny in the loss of chromosomes. To know what is unusual in (possibly) abnormal chromosome behavior in polyploids, it is critical to understand normal chromosome behavior. Surprisingly, little information is available on microtubule and centrosome behavior in early development of oysters and especially Crassostrea virginica. The objectives of this work were to document early development starting with meiosis in newly fertilized eggs through the first and second cleavage divisions, including centrosome inheritance and duplication observed by confocal microscopy.

Several studies have made cytological observations on early development in bivalves and can be roughly divided into two purposes. The first type concerns genetic improvement or chromosome manipulation, including polyploid induction, for aquaculture. Especially, for triploid production, which has high commercial value because of reproductive sterility, numerous studies have demonstrated meiotic and early zygotic development (Komaru et al. 1990b, Longo et al. 1993, Li et al. 2000) during chromosome set manipulation. Chromosome manipulation techniques include the induction of polyploidy with chemical reagents, such as, cytochalasin $\mathrm{B}$ and inactivation of chromosomes using UV irradiation (Longo et al. 1993, Li et al.

*Corresponding author. E-mail: ska@vims.edu DOI: $10.2983 / 035.036 .0319$
2000). The main target of chromosome manipulation in these studies was inhibition of polar body (PB) formation or first cleavage division. Most of these studies observed and described chromosome movements using normal light (Crassostrea virginica: Longwell \& Stiles 1968), electron microscopy (Crassostrea gigas: Longo et al. 1993), or epifluorescence microscopy (Pinctada fucata: Komaru et al. 1990a, 1990b; Chlamys nobilis: Komaru \& Wada 1991; C. gigas: Li et al. 2000). Work of this nature has contributed to the production of triploid scallops, clams, and mussels - some on at least a semicommercial scale (Beaumont \& Fairbrother 1991, Nell 2002).

The second type of cytological study of early development in bivalves concerns investigations in developmental biology and/or cytology. Marine bivalves are excellent candidates for meiotic and early zygotic studies (Guo \& Allen 1997) as reported by Longo (1972), Guo et al. (1992a, 1992b), and Longo et al. (1993). The main advantage of this system, compared with insect and mammalian ones, is that marine bivalves have high fecundities and practice external fertilization. For example, a 2-y-old female Pacific oyster, Crassostrea gigas, may produce 20-50 million eggs (Guo et al. 2009). Eggs from bivalves, in general, are smaller and contain less yolk material than eggs from fish and amphibians. Moreover, because meiosis is arrested at prophase I in the follicle, meiosis I and II can both be visualized in mature bivalve eggs after controlled fertilization. For example, Kuriyama et al. (1986) showed the microtubule cycle in oocytes of Spisula solidissima using fluorescence microscopy and immunological methods.

Surprisingly, few studies have been done on the meiotic and mitotic chromosome behavior in Crassostrea spp. Longwell and Stiles $(1968,1973)$ reported chromosome behavior stained by aceto-orcein at fertilization, meiosis, and early cleavage in Crassostrea virginica using optical microscopy. Li et al. (2000) compared nuclear behavior between normal and gynogenetic 
eggs of Crassostrea gigas using DAPI staining and epifluorescence microscopy. Longo et al. (1993) showed the course of fertilization and meiosis in CB-treated and untreated C. gigas eggs using electron microscopy and immunofluorescent techniques for the observation of DNA and microtubule organization, only from meiosis through metaphase of mitosis I. They discussed the behavior of centrosomes based on the organization of microtubules in the chromosome assemblages. Actual centrosomal behavior, however, has not been reported and there is little information for meiosis and even less for early cleavage. More contemporary techniques allow detailed visualization of complete meiotic and mitotic cytological structure and organelles. For example, Pielak et al. (2003, 2004, 2005) were able to elucidate the interaction between microtubule asters and cortical actin in Spisula solidissima oocytes using confocal microscopy. Several studies showed localization of centrosomes and nucleolinus in $S$. solidissima oocytes using confocal microscopy with in situ hybridization (Alliegro \& Alliegro 2005, 2008, 2013, Alliegro et al. 2006, 2010, 2012). Confocal microscopy affords exceptional clarity into the cytology of development. In this article, meiotic and early zygotic developments were documented in normal diploid $C$. virginica with confocal microscopy to document normal development.

\section{MATERIALS AND METHODS}

\section{Crosses Experiment and Sampling}

Two sets of pair matings were produced in the hatchery on August 9 (Cohort 1) and August 16 (Cohort 2), 2016. Ripe oysters from line DEBY.14 and hANA.14 held by Aquaculture Genetics and Breeding Technology Center were shucked and gonad biopsies were examined microscopically to determine the sex. The best specimens (maturity and fecundity) were used to make experimental crosses. Eggs for cohort 1 and 2 were stripspawned (Allen \& Bushek 1992) from hANA females. Males were from the DEBY.14 line for cross 1 and hANA.14 for the cohort 2. Eggs were counted before fertilization to obtain appropriate densities of eggs in the beakers. Both cohorts were performed at $24.5^{\circ}$ in 14.1 psu-filtered seawater.

Samples were fixed at $0,10,30,60,80$, and $100 \mathrm{~min}$ postfertilization, with the first three samples corresponding to periods of meiosis and the last three-mitosis. Oyster eggs are arrested before meiosis I and commence after fertilization. An appropriate volume of eggs was taken to assure at least 20,000 eggs were fixed.

\section{Sample Preparation for Confocal Microscopy}

\section{Fixation of Eggs and Staining}

Eggs sampled from beakers were screened through a $20-\mu \mathrm{m}$ Nytex screen to remove cell debris and placed into a $15-\mathrm{mL}$ Falcon tube containing $12 \mathrm{~mL}$ of fixative consisting of $0.1 \%$ TritonX-100, 4\% formaldehyde in PEM (100-mM PIPES, 5-mM EGTA, 1-mM MgCl $2, \mathrm{pH} 6.8$, using $\mathrm{NaOH}$ ). Eggs were simultaneously permeabilized and fixed in this fixative for $2 \mathrm{~h}$. After fixation, the samples were rinsed three times with $10-\mathrm{mM}$ glycine-phosphatase buffered saline (glycine-PBS pH7.4) and then washed with $10-\mathrm{mM}$ glycine-PBS twice. The samples were stored in 10-mM PBS (without glycine) in 15-ml falcon tube and stored at $4^{\circ}$ until they were used for cytoskeletal labeling.
For microtubules and centrosomes, before staining, fixed eggs were incubated for $1 \mathrm{~h}$ in $3 \%$ bovine serum albumin (BSA)/ PBS. Eggs were then washed once with glycine-PBS. Afterward, eggs were washed again, this time with $0.1 \% \mathrm{BSA} / \mathrm{PBS}$ (containing $0.1 \%$ TritonX). For staining, either of two primary antibodies was used, specific for either microtubules or centrosomes, in combination with a secondary antibody containing the fluorochrome. Microtubules were labeled with mouse monoclonal anti- $\alpha$ tubulin (Sigma-T9026) diluted to $0.5 \mu \mathrm{L} / \mathrm{mL}$ by $1 \% \mathrm{BSA} / \mathrm{PBS}$ (containing $0.1 \%$ TritonX) by incubating for $24 \mathrm{~h}$ at room temperature. Centrosomes were labeled with mouse monoclonal anti- $\gamma$ tubulin (Sigma-T6557) diluted to $0.5 \mu \mathrm{L} / \mathrm{mL}$ by $1 \%$ BSA/PBS (containing $0.1 \%$ TritonX) as the primary antibody by incubating for $48 \mathrm{~h}$ at room temperature. After incubation, the eggs were washed three times with $0.1 \% \mathrm{BSA} / \mathrm{PBS}$ (containing $0.1 \%$ TritonX).

Eggs stained for microtubules or for centrosomes were labeled with the goat antimouse $\operatorname{IgG}(\mathrm{H}+\mathrm{L})$ secondary antibody pretagged with Alexa Fluor 488 conjugate diluted to $5 \mu \mathrm{L} / \mathrm{mL}$ by $1 \%$ BSA/PBS (containing $0.1 \%$ TritonX). The eggs were washed three times with $0.1 \% \mathrm{BSA} / \mathrm{PBS}$ (containing $0.1 \%$ TritonX).

For chromosomes and nuclei, eggs labeled for either microtubules or for centrosomes were then counterstained with DAPI stock solution (DAPI $5 \mu \mathrm{g} / \mathrm{mL}, 2$-mercapt-ethylamine hydrochloride $10 \mathrm{mM}$, Tris $10 \mathrm{mM}$, EDTA-2Na $100 \mathrm{mM}, \mathrm{NaCl}$ $100 \mathrm{mM}$ ) diluted to $0.5 \mu \mathrm{g} / \mathrm{mL}$ by $1 \% \mathrm{BSA} / \mathrm{PBS}$ (containing $0.1 \%$ TritonX) and incubated for $20 \mathrm{~min}$ to visualize the chromosomes. The labeled samples were washed twice with $0.1 \% \mathrm{BSA} / \mathrm{PBS}$ (containing $0.1 \%$ TritonX).

For mounting slides permanently, after removal of most of the supernatant, a suspension of eggs was added to about $60 \mu \mathrm{L}$ of mounting medium (ProLong Gold Antifade Mountant) on the slide glass (Matunami Glass Ind., Ltd). The specific slide glasses were coated around the edges and uncoated in the center to avoid crushing the eggs when the cover slip was mounted (Alliegro et al. 2010). Eggs and mounting medium were aspirated gently to mix the eggs and medium. A cover slip was placed over the sample/mounting medium mixture and the edges sealed with nail polish.

Samples were viewed with a standard Olympus epifluorescence microscope and by confocal microscopy using an Olympus Laser Scanning Fluoview FV1200 with image processing via FV10-ASW 4.2 Viewer (Olympus). Images are maximum intensity projections of the entire z-stack. In addition, an optical microscope BX-51(Olympus) using cellSens (Olympus) image analysis software was used for estimating egg diameter.

\section{Measurements}

Egg diameter was determined under $100 \times$ magnification in fixed eggs by taking four diameter readings at each egg. Eggs in which the maximum egg diameter differed from a minimum egg diameter by more than $10 \mu \mathrm{m}$ were not analyzed because of their probable immaturity. Average egg diameter from each diploid female was statistically analyzed with a Welch's $t$-test at $P=0.05$.

Spindle size was defined as the distance between two centrosomes. Measurement of spindle size was determined on a photograph and measured using imaging software FV10ASW 4.2 Viewer. In meiosis I, the images were taken with the following three conditions: (1) Developmental stage was 
meiosis I metaphase. (2) The peripheral centrosome was attached to the egg cortex. (3) The images from the confocal were taken with the 2D-mode and the two centrosomes had to be in the same section. The thickness of single optical section in the 2D-mode was theoretically $1.34 \mu \mathrm{m}$. For measuring the first mitotic division (MT1), the distance between centrosomes was determined under following two conditions: (1) Developmental stage was metaphase of MT1. (2) The images were taken by the 2D-mode with confocal microscopy and two centrosomes were in the same section.

\section{RESULTS}

\section{Egg Morphology}

The overall morphology of eggs obtained from stripped follicles after incubation in seawater for $30 \mathrm{~min}$ took two forms: (1) Eggs were round (Fig. 1A) or (2) eggs were teardrop shaped (Fig. 1B). Uneven distribution of egg yolk was observed in both forms with optical microscopy. Egg diameter was $47.0 \pm 1.9 \mu \mathrm{m}$ $(n=73)$ for the first cohort and $49.1 \pm 1.8 \mu \mathrm{m}(n=93)$ for the
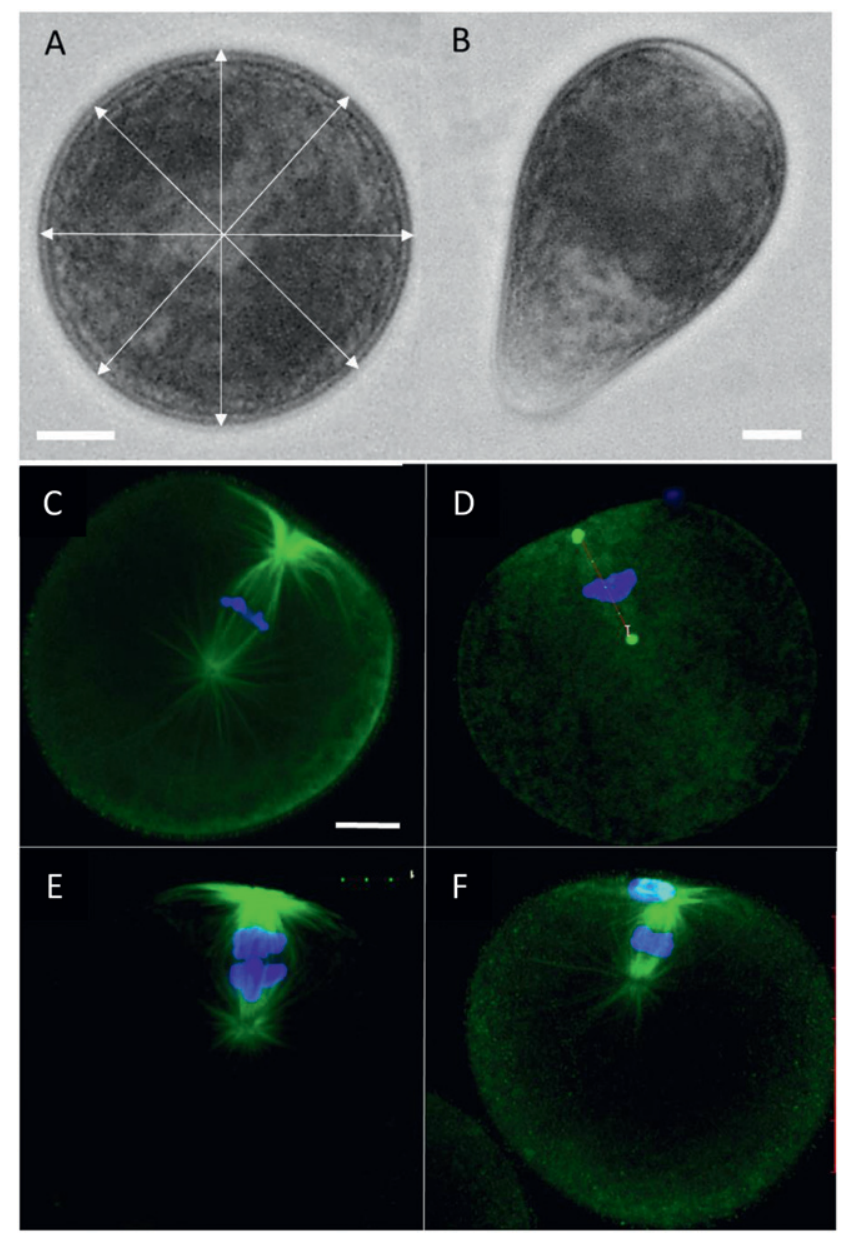

Figure 1. Oocytes $30 \mathrm{~min}$ after strip spawning (A, B) observed by light microscopy and newly fertilized eggs of Crassostrea virnginica by fluorescence microscopy (C-F). Microtubules (C, E, F) and centrosomes (D) were labeled with $\alpha$ - or $\gamma$-tubulin antibody, respectively. Chromosomes were located on the metaphase plate of first meiosis (C, D). (E) Early anaphase of first meiosis. (F) Metaphase of second meiosis. The first polar body was on the egg surface. Scale bar $=10 \mu \mathrm{m}$. second, which was significantly different $(P<0.001)$ between the two diploid females used in the cohort. Teardrop-shaped eggs rounded up after fertilization.

\section{Meiosis}

Microtubules and centrosomes were visualized by immunostaining of the eggs and maternal and paternal chromosomes with DAPI staining. A spindle was formed by two centrosomes and microtubules spread radially from each centrosome. The meiotic spindles were located near the animal pole. Peripheral astral microtubules were oriented near the animal pole and spread along the cell cortex (Fig. 1C). Central astral microtubules were spread radially at the center of the egg. In most eggs, one centrosome was attached to the cortex of oocytes and the other centrosome was located centrally. The average distance between the two centrosomes was $14.4 \pm 1.3 \mu \mathrm{m}(n=50)$ for the first cross and $13.9 \pm 1.2 \mu \mathrm{m}(n=50)$ for the second. There was a significant difference between the two crosses, i.e., egg source $(P=0.031)$. Chromosomes derived from the egg were arranged along the equatorial plane of the meiotic spindle. Karyokinesis was observed to proceed perpendicular to the egg surface (Fig. 1D, E). After extrusion of the first polar body (PB1), the second meiotic spindle appeared. During the transition from the first to the second meiotic division, the maternal chromosomes never appeared in a decondensed state. At sampling periods immediately after cytokinesis, chromosomes were observed at the start of second meiosis. When the second meiotic spindle appeared, it was not always perpendicular to the egg surface, but later was observed perpendicular to the egg surface for the release of second polar body (PB2) (Fig. 1F). Extruded PB1 and PB2 were very close together. In some cases, the number of polar bodies on the egg surface could not be counted accurately.

\section{Male Pronucleus Transformation and Centrosome Inheritance}

Just after insemination, the sperm nuclei in the cytoplasm of eggs were observed still in a condensed (Fig. 2A) state. Sperm could be seen at various locations around the perimeter of the egg. The centrosome was not observed at the periphery of the sperm nucleus at early metaphase II (Fig. 2A). At meiosis II, one centrosome was observed beside the sperm nucleus (Fig. 2B). The centrosome originating from the sperm duplicated by late anaphase of meiosis II (Fig. 2C). When the release of the PB2 was complete, the sperm nucleus began to swell (Fig. 2D), with two centrosomes still associated with it. The female pronucleus also swelled, but the centrosomes associated with the female pronucleus during meiosis disappeared after PB2 formation. At this time, duplicated centrosomes migrated to opposite sides of the male pronucleus (Fig. 2E). The network of microtubules began to build in the egg cytoplasm (not shown, but could be observed with microtubule staining). Syngamy occurred as the swollen male and female pronuclei combined in the center of the egg, with the two centrosomes derived from the male pronuclei (Fig. 2F). Intermingled chromosomes began to condense and line up on the equatorial plane for first mitosis (Figs. 2G and $3 \mathrm{H}$ ).

\section{Mitosis—First Cleavage and Mitotic Centrosome Duplication}

Chromosomes from the male and female pronuclei gathered and aligned on the equatorial plane of the spindle organized by 


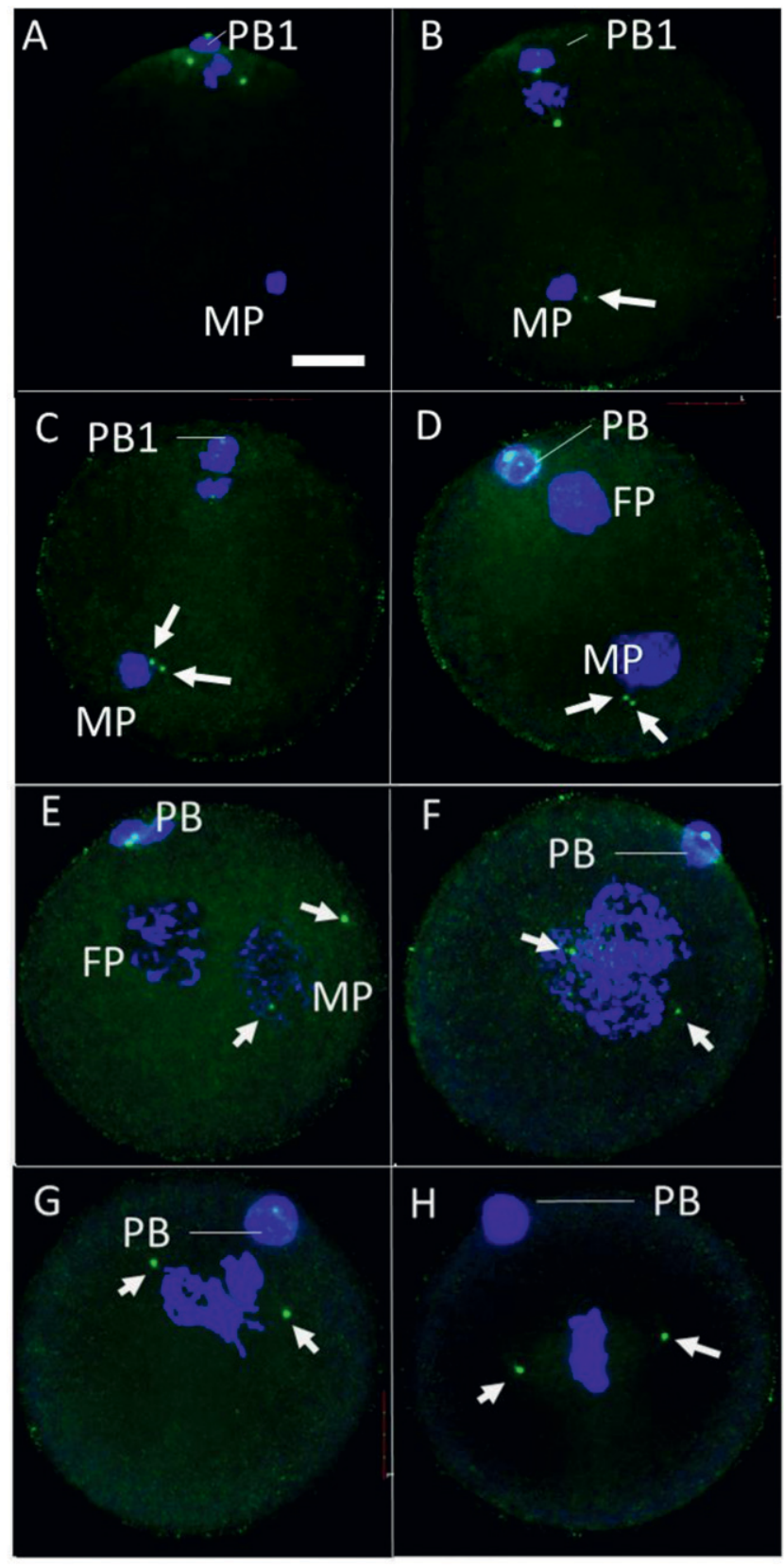

Figure 2. Behavior of pronuclei and centrosome inheritance in fertilized eggs of Crassostrea virginica. (A) At metaphase of meiosis II, male pronucleus (MP) is condensed and has only one centrosome (B). (C) By late anaphase of meiosis II, the centrosome of the MP has duplicated. Male pronucleus and female pronucleus (FP) begin to swell. (D) They migrate to cell center as duplicated centrosomes migrate to opposite sides of MP (E). (F) Syngamy precedes condensation of chromosomes from MP and FP (G). (H) Finally, chromosomes organize on the metaphase plate of MT1. White arrows indicate centrosomes. Scale bar $=10 \mu \mathrm{m}$.

the two centrosomes (Fig. 3A). The spindle was organized in the center of the egg. The spindle axis of the first cleavage formed perpendicular to the axis of the animal-vegetal pole. At metaphase of MT1, the average distance between the two centrosomes was $17.56 \pm 1.9 \mu \mathrm{m}(n=10)$ for the first cohort and 15.9 \pm $1.0 \mu \mathrm{m}(n=11)$ for the second, with a significant difference $(P=$ $0.045)$ between the two cohorts. Beginning at anaphase of MT1,

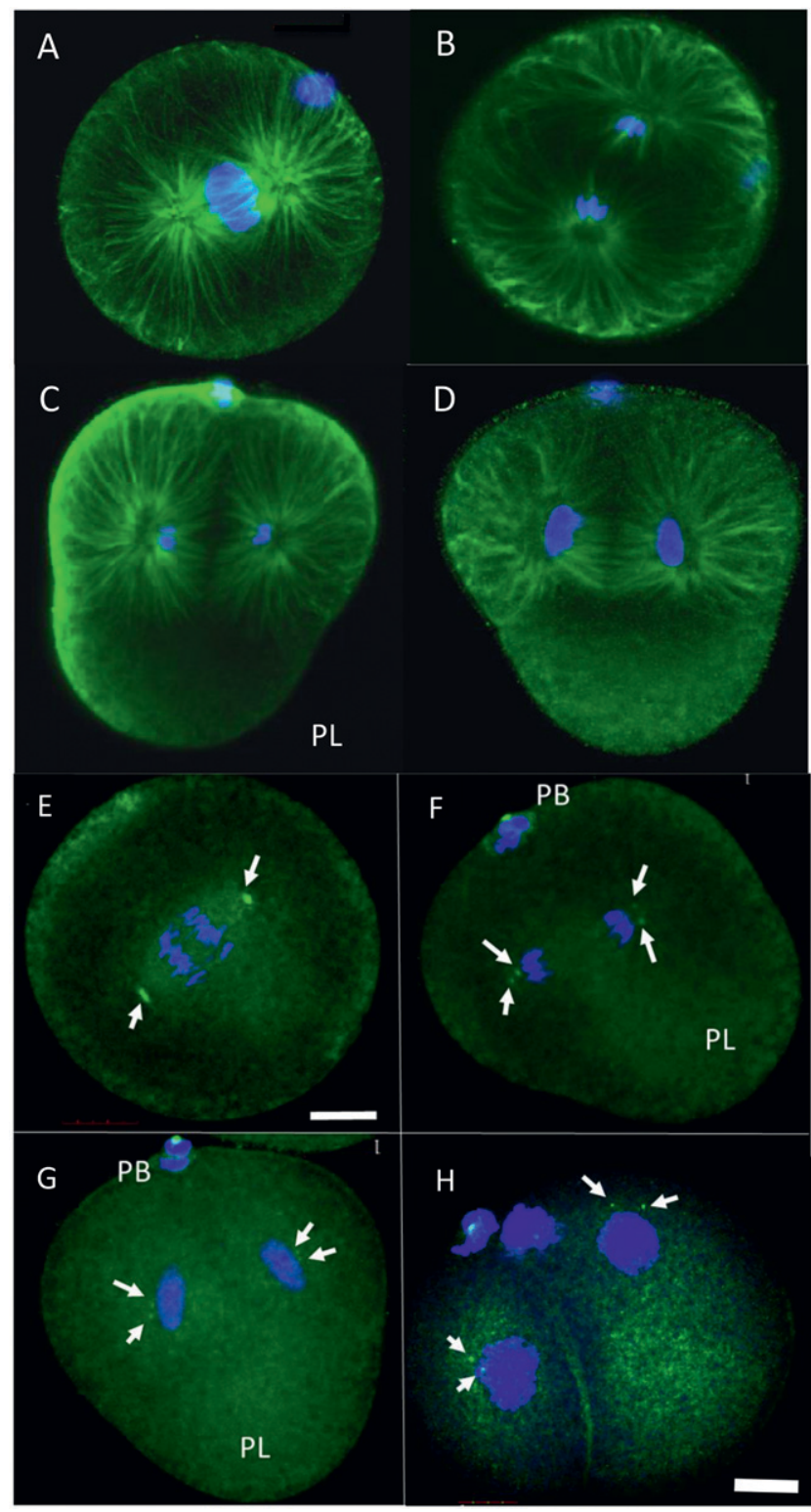

Figure 3. First cleavage of mitosis in dividing eggs of Crassostrea virginica. (A) In early anaphase of MT1, microtubule structure can be clearly seen to have organized around the centrosomes. (B) Centrosomes are indistinct by anaphase. (C, D) By late anaphase, PL has formed, but is absorbed later. (D) Early midbody is clearly visible at telophase of mitosis 1 and shrinks with progression of cell cleavage. (E) Mitotic centrosomes at early anaphase of first cleavage (arrows). (F) Mitotic centrosomes (arrows) at disengagement at late anaphase of first cleavage. (G) Telophase of first cleavage. Condensed chromosomes had segregated to each spindle pole. After migration of chromosomes, the signal of $\gamma$-tubulin became two. Condensed chromosome started to decondense at telophase. (H) Nuclei were observed in a swollen state in early two cell stage. White arrows indicate centrosomes.

the fluorescent signal of the centrosome diminished, probably because the centrosome was undergoing duplication. Two fluorescent signals were recognized at each pole of the spindle (Figs. 3B and 4C). Also, at late anaphase of MT1, the polar lobe (PL) formed at the vegetal pole (Figs. 3C and 4D) and was later absorbed into one of the two cells of the first cleavage to form large and small blastomeres. Cytokinesis was observed at times 
synchronous to the absorption of the PL. The cleavage furrow was colocated with the position of the extruded polar bodies at the animal pole. Also, the midbody between the cells became apparent at telophase (Fig. 3D), apparently decreasing with the contraction of the contractile ring.

At early anaphase of MT1 (Fig. 3E), centrosomes were distinct, one at each pole of the spindle. The centrosomes were elongated. But by late anaphase, they had begun to duplicate and were observed as two distinct signals at each pole (Fig. 3F). By telophase, segregated chromosomes had started to decondense (Fig. 3G) with duplicated centrosomes.

\section{Two-Cell}

After the formation of two cells (Fig. 3H), nuclei swelled and chromatin in the nuclei was observed in a more condensed state. A network of microtubules was evident throughout the egg cytoplasm at this stage (Fig. 4A). The astral microtubules that had nucleated from each centrosome were duplicated in late anaphase of MT1 and had migrated to opposite poles of their respective nuclear masses and started to form the spindle poles (Fig. 4B). At metaphase of MT1, chromosomes segregated after aligning on the equatorial plane of each spindle. This seemed to occur simultaneously in both blastomeres (Fig. 4C). The second karyokinesis of the two blastomeres, however, was asynchronous in the vast majority of observations (Fig. 4D). In the eggs observed that were asynchronous, chromosome segregation in the large blastomere was seen before those in the small blastomere. The PL again formed and was then absorbed in the large blastomere at anaphase. At the telophase of the second mitotic division (MT2), the width of the midbody that formed between each blastomere decreased with the contraction of the contractile ring as in the first cleavage.

\section{Four-Cell}

The blastomere that absorbed the PL became the large blastomere (Fig. 4E). The other cells appeared to be of equal size. Chromosome decondensation occurred simultaneously in all four cells (Fig. 4F), whereas a network of microtubules was built in the cytoplasm, as observed in former stages. The large blastomere was the first to form the next mitotic spindle in the four-cell stage (Fig. $4 \mathrm{G})$. As the mitotic apparatus of the large blastomere became organized with chromosomes at the metaphase plate, the chromosomes of the three small blastomeres were less condensed and the aster not completely segregated. Figure 4D shows the four-cell stage. In the large blastomere, centrosomes were already duplicated at the poles for the next division. In the small blastomeres, the centrosomes were observed as one unit located on both sides of the condensed chromosomes, but not on the metaphase plates. Thus, at the four cell stage, asynchrony was obvious.

\section{DISCUSSION}

\section{Egg Structure at Fertilization}

In this study, eggs were obtained by strip spawning (Allen \& Bushek 1992), which is an increasingly common way for research and commercial spawning of oyster species. Other studies have documented early development of oyster eggs obtained by strip spawning. Longo et al. (1993) documented two morphologies of Crassostrea gigas fertilized egg from strip
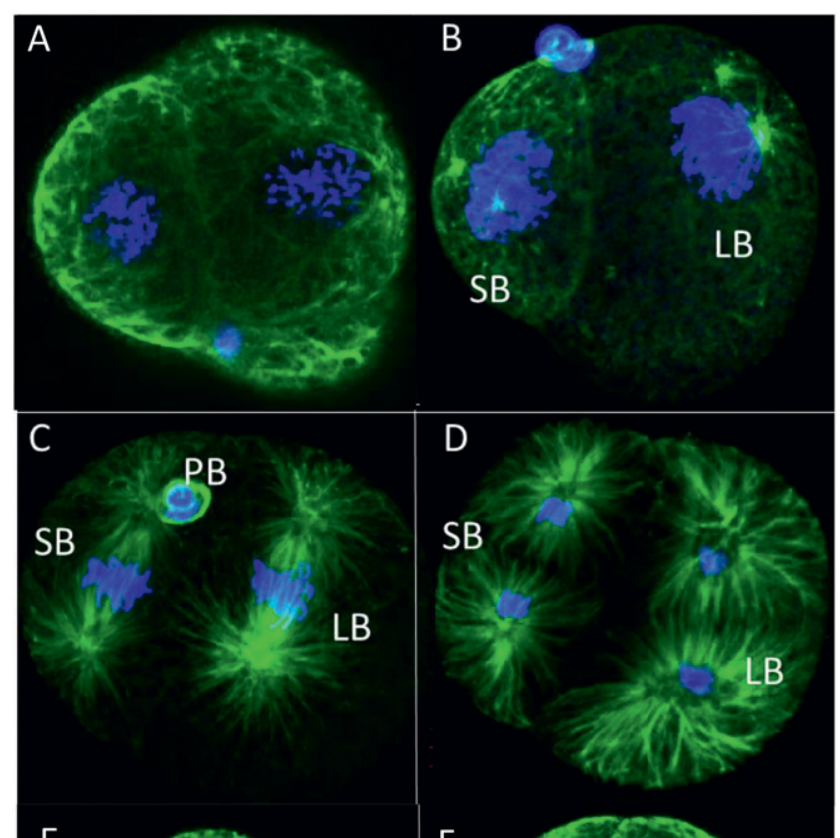

SB
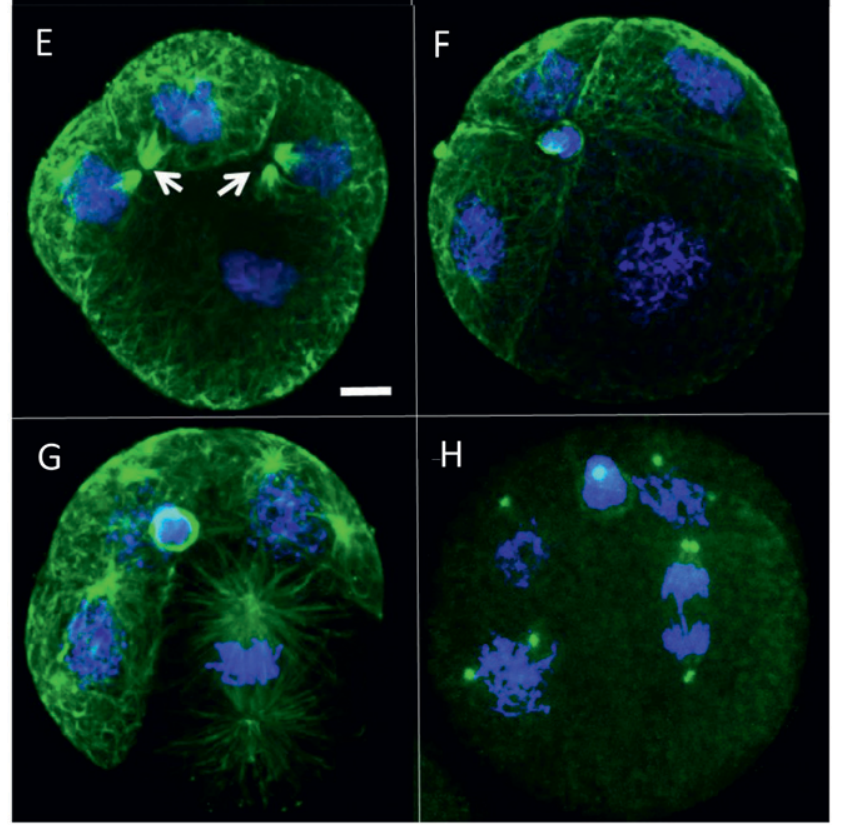

Figure 4. Two-cell and 4-cell stage in Crassostrea virginica embryos. (A) Chromosomes under duplication in each cell. (B) Centrosome migrated to opposite poles and began to form spindles. (C) Metaphase occurred simultaneously in both cells, but mitosis was usually advanced in the large blastomere (D). The microtubules (E-G) and centrosomes (H) were labeled with $\alpha$ - or $\gamma$-tubulin antibody, respectively. (E) Midbodies (arrows) were clearly visible as cells divided and nuclei entered $\mathrm{S}$ phase. (F) All four blastomeres entered $\mathrm{S}$ phase simultaneously. (G) Large blastomere began mitosis first. (H) Chromosome segregation was completed in the large blastomere first. Scale bar $=10 \mu \mathrm{m}$.

spawning. The significance of these two forms with respect to gamete maturation, fertilizability, and potential for development was unclear. It was assumed that teardrop-shaped eggs were immature, having been torn from their follicle walls where they were still attached. Such eggs generally round up after time and are equally fertilizable, but this has not been tested directly. Nonetheless, egg diameter was measured only on round eggs. 
On average between the cohorts, the diameter of eggs from diploid Crassostrea virginica was $48.2 \mu \mathrm{m}$. The egg size differing significantly from each other (female from first cohort versus female from second) was surprising because the egg size of $C$. virginica is highly regulated, undergoing constant balancing selection between fecundity and nourishment (Powell et al. 2011). At the same time, eggs from the two cohorts came from two different highly selected lines and the effect of domestication on the egg size is unknown.

\section{Meiosis}

Meiotic maturation in molluscan species is characterized by two general types: eggs with a secondary arrest in the first meiotic metaphase or eggs with no secondary arrest. In some bivalves, such as Spisula or Barnea, spawned oocytes are arrested at prophase I. Fertilization occurs at this stage leading to reinitiation of meiosis (Dubé \& Guerrier 1982, Colas \& Dubé 1998). By contrast, bivalves, such as Ruditapes and Crassostrea (Kuraishi \& Osanai 1988), undergo germinal vesicle breakdown after spawning and are then arrested at metaphase of meiosis I. The Crassostrea virginica eggs in this study were incubated for at least $30 \mathrm{~min}$ after strip spawning, and most of the eggs were arrested at metaphase of meiosis I. In some cases, the meiotic spindle pole was unattached to the cortex of the animal pole (Fig. 2A). It seemed clear that the spindle migrates to the animal pole eventually. Kuriyama et al. (1986) and Pielak et al. (2003) described similar meiotic spindle behavior using Spisula oocytes. Longo et al. (1993) also documented spindle formation during meiosis in Crassostrea gigas. Interestingly, Longo et al. (1993) proposed that this spindle migration in bivalves can occur without microfilaments.

The male pronucleus condensed and remained quiescent until PB2 extrusion in the egg cytoplasm, at which point it began to swell. Similar findings were reported in previous work (Crassostrea gigas: Longo et al. 1993, Stephano \& Gould 2000, Asterina miniata: Stephano \& Gould 2000; Corbicula sandai and Corbicula fluminea: Obata et al. 2006). On the other hand, duplicated centrosomes were observed at late anaphase of meiosis II (Fig. 3C). The paternal centrosome may have been duplicated at the same time as PB2 extrusion or before. Stephano and Gould (2000) proposed that MAP kinase is involved in sperm centrosome suppression based on the observation for microtubule organization in which MAP kinase is inhibited during meiosis in oyster eggs.

\section{Pronuclei and Centrosome Inheritance}

Longo et al. (1993) investigated the course of fertilization events in CB-treated Crassostrea gigas eggs using electron microscopy and immunofluorescent techniques for the observation of DNA and microtubule organization. Based on microtubule formation, they suggest that centrosomes of the embryo of C. gigas are paternally inherited (Longo et al. 1993, Longo 1997). Actual centrosome behavior was not reported, however. Observations from this study followed the behavior of centrosomes from metaphase of meiosis I through metaphase I and clearly showed that centrosomes are inherited from sperm whereas maternal centrosomes disappear (Fig. 2D). Therefore, it is evident from this study that two centrosomes derived from spermatozoa act as microtubules organization center of MT1. More correctly, the one centrosome derived from sperm duplicated just after PB1 extrusion. If oocytes were to retain a fully functional maternal centrosome capable of replication, introduction of a paternal centrosome would result in the presence of two replication-competent centrosomes and lead to the formation of tetrapolar spindles and disruption of chromosome segregation or aneuploidy (Palazzo et al. 1999). Thus, the ability of the maternal centrosome to replicate seems lost during the completion of meiosis, leaving the paternal centrosome to replicate as needed for coordinated development (Sluder et al. 1989, Schatten 1994).

In oocytes of starfish, Asterina pectinifera and the clams, Spisula solidissima and Corbicula sandai, the ability of the maternal centrosome to replicate is selectively destroyed during meiosis II (Kuriyama et al. 1986, Sluder et al. 1989, 1993, Obata et al. 2006, Borrego-Pinto et al. 2016). Importantly, in both Asterina and Spisula, the male centrosome is present in the egg cytoplasm when the ability of the maternal centrosome to replicate and to nucleate microtubules was destroyed (Sluder et al. 1989, 1993, Wu \& Palazzo 1999). Yet, despite the deactivation of the maternal centrosome, maternal components are still required for restoration of the zygotic centrosome during organization of microtubules (Schatten 1994).

\section{Mitosis}

Normal cell division proceeds through the linkage of the cell cycle, the nuclear cycle, and the centrosome cycle in animal cells (Doxsey et al. 2005). The first two mitotic divisions were observed in this study. Although cell number was different, centrosomes and cytoplasmic structure exhibited similar behavior in both MT1 and MT2 in accordance with the nuclear cycle. Furthermore, the microtubule cycle was closely related to the centrosome cycle because centrosomes coordinate microtubule organization. The following description presents the linkage of nuclear cycle, cell cycle, and centrosome cycle in first two mitotic divisions of Crassostrea virginica zygotes.

\section{Metaphase to Anaphase}

The duplicated genome must be divided into two daughter cells by the spindle apparatus that is organized by centrosomes. Centrosomes are composed of two orthogonally arranged centrioles surrounded by an amorphous mass of protein, termed the pericentriolar material (Nigg 2002). One centrosome with two centrioles performs as a single microtubule organization center (Nigg 2002, 2007). In the initial step for Crassostrea virginica, chromosomes condensed and aligned on the equatorial plane of the spindle that was formed by microtubules radiating from the two centrosomes at metaphase. Chromosome segregation began after all chromosomes were aligned on the equatorial plane. Normally, the process is regulated by a spindle assembly checkpoint that monitors attachment of the spindle microtubules to kinetochores of chromosomes (Doxsey et al. 2005). Changes in cell structure and centrosome number during the metaphase to anaphase stage were not observed by us.

\section{Late Anaphase}

There were several changes in both cell structure and centrosome behavior in late anaphase of the two mitotic divisions. For the centrosome, although a single centrosome was observed at each pole at early anaphase of MT1, two distinct signals of $\gamma$-tubulin were recognized in each spindle pole at late anaphase. In more detail, these duplicated signals of $\gamma$-tubulin were 
diminished compared with the signal in early anaphase of MT1. A similar finding was observed in MT2 (data not shown). The interpretation was that the centrosomes were either disengaged before or undergoing duplication. This distinction was not possible because of the limitations of gamma tubulin staining. To undergo duplication, the orthogonal association of centrioles is lost and the amount of $\gamma$-tubulin decreases by about one-third to one-fifth. The dissociation is accompanied by a striking decrease in microtubule nucleating activity in centrosomes (Khodjakov \& Rieder 2001). This process is the first step for centrosome duplication (Meraldi \& Nigg 2002). In Crassostrea virginica, centrosome duplication began at late anaphase of MT1. Furthermore, change of cell structure also started at late anaphase of early mitotic division. For MT1, the PL protruded from vegetal pole. For the second cleavage, the PL extruded from the large blastomere (data not shown). Dorresteijn (1990) reported that spiralian embryos, such as mollusca with an unequal first mitotic division, can be divided into two groups: with or without PL formation. Polar lobe formation has been observed in other species during early development (e.g., Crassostrea gigas: Deno 1998; Crepidula fornicata: Henry et al. 2006). In embryos without a PL, the first mitotic division shifts to one side and one spindle pole attaches to the cortex (e.g., Dreissena polymorpha: Luetjens \& Dorresteijn 1998; Corbicula sandai: Obata et al. 2006). On the contrary, the spindle of the first cleavage is oriented in the center of the zygote. This is the typical pattern of the first cleavage with PL formation.

\section{Telophase to Prophase}

In Crassostrea virginica, the centrosomes organized the microtubules from metaphase to late anaphase to establish the spindle apparatus. The fluorescence of the astral formation diminished when the chromosomes decondensed and formed the nucleus. This loss of $\gamma$-tubulin signal is probably associated with dissassembly of microtubules at late anaphase and seemed to be related to the centrosome cycle. $\gamma$-tubulins are essential for nucleation of microtubules (Palazzo et al. 1999). The main feature of telophase was cytoplasmic division. The cleavage furrow was observed when segregated chromosomes decondensed and formed nuclei. Moreover, the midbody was observed between the two dividing cells at the end of cytoplasmic division. The midbody is common and synchronous with the formation of the contractile ring in animal cells (Steigemann \& Gerlich 2009). At this time, synchrony was observed between cytoplasmic division and PL reabsorption. In contrast to this dynamic cell structural change, the two duplicated centrosomes apparently kept their position and structure. Important centrosomal behavior was observed when cell division was completed: two adjacent centrosomes separated and acted as microtubule organizing centers. Centrosome separation is known to be caused by the action of microtubules-dependent motor proteins, such as Eg5 (Smith et al. 2011). The two separated centrosomes formed a bipolar spindle and contributed to chromosome segregation. Asynchrony of the cell cycle in each cell at four-cell stage was also observed. Even though cell number and cell structure are different between first and second cell division, the cell cycle, nuclear cycle, and centrosome cycle are coordinated in both cell divisions.

In this article, detailed observations on meiotic and early zygotic development were presented. The results, obtained by double staining nuclear and microtubules or centrosomes clearly showed linkage of cell cycle, nuclear cycle, and centrosome cycle. This fundamental knowledge will be useful not only to understand the detailed mechanism of meiosis and early mitosis in the oyster, but also for future investigations into meiotic and mitotic abnormalities in polyploids of Crassostrea virginica.

\section{LITERATURE CITED}

Allen, S. K. \& D. Bushek. 1992. Large-scale production of triploid oysters, Crassostrea virginica (Gmelin), using "stripped" gametes. Aquaculture 103:241-251.

Allen, S. K., Jr., X. Guo, G. Burreson \& R. Mann. 1996. Heteroploid mosaics and reversion among triploid oysters, Crassostrea gigas: fact or artifact. J. Shellfish Res. 18:293.

Alliegro, M. A., J. J. Henry \& M. C. Alliegro. 2010. Rediscovery of the nucleolinus, a dynamic RNA-rich organelle associated with the nucleolus, spindle, and centrosomes. Proc. Natl. Acad. Sci. USA 107:13718-13723.

Alliegro, M. C. \& M. A. Alliegro. 2005. Differential expression of tyrosinated tubulin in Spisula solidissima polar bodies. Dev. Dyn. 232:216-220.

Alliegro, M. C. \& M. A. Alliegro. 2008. Centrosomal RNA correlates with intron-poor nuclear genes in Spisula oocytes. Proc. Natl. Acad. Sci. USA 105:6993-6997.

Alliegro, M. C. \& M. A. Alliegro. 2013. Localization of rRNA transcribed spacer domains in the nucleolinus and maternal procentrosomes of surf clam (Spisula) oocytes. RNA Biol. 10:391-396.

Alliegro, M. C., M. A. Alliegro \& R. E. Palazzo. 2006. Centrosomeassociated RNA in surf clam oocytes. Proc. Natl. Acad. Sci. USA 103:9034-9038.

Alliegro, M. C., S. Hartson \& M. A. Alliegro. 2012. Composition and dynamics of the nucleolinus, a link between the nucleolus and cell division apparatus in surf clam (Spisula) oocytes. J. Biol. Chem. 287:6702-6713.
Beaumont, A. R. \& J. E. Fairbrother. 1991. Ploidy manipulation in molluscan shellfish: a review. J. Shellfish Res. 10:1-18.

Borrego-Pinto, J., K. Smogyi, M. A. Karreman, J. König, T. MüllerReichert, M. Bettencourt-Dias, P. Gönczy, Y. Schwab \& P. Lénárt. 2016. Distinct mechanisms eliminate mother and daughter centrioles in meiosis of starfish oocytes. J. Cell Biol. 212:815-827.

Colas, P. \& F. Dubé. 1998. Meiotic maturation in mollusc oocytes. Semin. Cell Dev. Biol. 9:539-548.

Deno, T. 1998. Autonomous fluorescence localized at the polar lobe of eggs of Japanese oyster, Crassostrea gigas. Bull. Osaka Kyoiku Univ. III Nat. Sci. 46:195-207.

de Sousa, J. T., S. K. Allen, Jr., H. Baker \& J. L. Matt. 2016. Aneuploid progeny of the American oyster, Crassostrea virginica, produced by tetraploid $\times$ diploid crosses: another example of chromosome instability in polyploid oysters. Genome 59:327-338.

de Sousa, J. T., D. Matias, S. Joaquim, R. Ben-Hamadou, \& A. Leitão. 2011. Growth variation in bivalves: new insights into growth, physiology and somatic aneuploidy in the carpet shell Ruditapes decussatus. J. Exp. Mar. Biol. Ecol. 406:46-53.

Dorresteijn, A. W. 1990. Quantitative analysis of cellular differentiation during early embryogenesis of Platynereis dumerilii. Roux's Arch. Dev. Biol. 199:14-30.

Doxsey, S., W. Zimmerman \& K. Mikule. 2005. Centrosome control of the cell cycle. Trends Cell Biol. 15:303-311. 
Dubé, F. \& P. Guerrier. 1982. Activation of Barnea candida (Mollusca, Pelecypoda) oocytes by sperm or $\mathrm{KCl}$, but not by $\mathrm{NH}_{4} \mathrm{Cl}$, requires a calcium influx. Dev. Biol. 92:408-417.

Guo, X. \& S. K. Allen, Jr. 1997. Sex and meiosis in autotetraploid Pacific oyster, Crassostrea gigas (Thunberg). Genome 40:397-405.

Guo, X., K. Cooper, W. K. Hershberger \& K. K. Chew. 1992a. Genetic consequences of blocking polar body I with cytochalasin B in fertilized eggs of the Pacific oyster, Crassostrea gigas: I. Ploidy of resultant embryos. Biol. Bull. 183:381-386.

Guo, X., W. K. Hershberger, K. Cooper \& K. K. Chew. 1992b. Genetic consequences of blocking polar body I with cytochalasin $\mathrm{B}$ in fertilized eggs of the Pacific oyster, Crassostrea gigas: II. Segregation of chromosomes. Biol. Bull. 183:387-393.

Guo, X., Y. Wang, Z. Xu \& H. Yang. 2009. Chromosome set manipulation in shellfish. In: Burnell, G. \& G. Allan, editors. New technologies in aquaculture: improving production efficiency, quality and environmental management. pp. 165-194. Sawston, Cambridge, UK: Woodhead Publishing.

Henry, J. Q., K. J. Perry \& M. Q. Martindale. 2006. Cell specification and the role of the polar lobe in the gastropod mollusc Crepidula fornicata. Dev. Biol. 297:295-307.

Khodjakov, A. \& C. L. Rieder. 2001. Centrosomes enhance the fidelity of cytokinesis in vertebrates and are required for cell cycle progression. J. Cell Biol. 153:237-242.

Komaru, A., K. Matsuda, T. Yamakawa \& K. Wada. 1990a. Meiosis and fertilization of the Japanese pearl oyster eggs at different temperature observed with a fluorescence microscope. Nippon Suisan Gakkaishi 56:425-430.

Komaru, A., K. Matsuda, T. Yamakawa \& K. Wada. 1990b. Chromosome behavior of meiosis-inhibited eggs with cytochalasin B in Japanese pearl oyster. Nippon Suisan Gakkaishi 56:1419-1422.

Komaru, A. \& K. Wada. 1991. Different processes of pronuclear events in pressure-treated and CB-treated zygotes at the second meiosis in scallop. Nippon Suisan Gakkaishi 57:1219-1223.

Kuraishi, R. \& K. Osanai. 1988. Behavior of sperm nuclei in meiotic eggs of the oyster, Crassostrea gigas. Bull. Mar. Biol. Stn. Asamushi Tohoku Univ. 18:57-65.

Kuriyama, R., G. G. Borisy \& Y. Masui. 1986. Microtubule cycles in oocytes of the surf clam, Spisula solidissima: an immunofluorescence study. Dev. Biol. 114:151-160.

Leitão, A., P. Boudry \& C. Thiriot-Quiévreux. 2001. Negative correlation between aneuploidy and growth in the Pacific oyster, Crassostrea gigas: ten years of evidence. Aquaculture 193:39-48.

Li, Q., M. Osada, M. Kashihara, K. Hirohashi \& A. Kijima. 2000. Cytological studies on artificially induced gynogenesis in the Pacific abalone. Fish. Sci. 66:701-707.

Longo, F. J. 1972. The effects of cytochalasin B on the events of fertilization in the surf clam, Spisula solidissima. I. Polar body formation. J. Exp. Zool. 182:321-344.

Longo, F. J. 1997. Fertilization, $2^{\text {nd }}$ edition. New York, NY: Garland Science.

Longo, F. J., L. Mathews \& D. Hedgecock. 1993. Morphogenesis of maternal and paternal genomes in fertilized oyster eggs (Crassostrea gigas): effects of cytochalasin B at different periods during meiotic maturation. Biol. Bull. 185:197-214.

Longwell, A. C. \& S. S. Stiles. 1968. Fertilization and completion of meiosis in spawned eggs of the American oyster, Crassostrea virginica Gmelin. Caryologia 21:65-73.

Longwell, A. C. \& S. S. Stiles. 1973. Gamete cross incompatibility and inbreeding in the commercial American oyster, Crassostrea virginica Gmelin. Cytologia (Tokyo) 38:521-533.
Luetjens, C. M. \& A. W. Dorresteijn. 1998. Dynamic changes of the microtubule system corresponding to the unequal and spiral cleavage modes in the embryo of the zebra mussel, Dreissena polymorpha (Mollusca, Bivalvia). Zygote 6:239-248.

McCombie, H., S. Lapègue, F. Cornette, C. Ledu \& P. Boudry. 2005. Chromosome loss in bi-parental progenies of tetraploid Pacific oyster Crassostrea gigas. Aquaculture 247:97-105.

Meraldi, P. \& E. A. Nigg. 2002. The centrosome cycle. FEBS Lett. 521:9-13.

Nell, J. A. 2002. Farming triploid oysters. Aquaculture 210:69-88.

Nigg, E. A. 2002. Centrosome aberrations: cause or consequence of cancer progression? Nat. Rev. Cancer 2:815-825.

Nigg, E. A. 2007. Centrosome duplication: of rules and licenses. Trends Cell Biol. 17:215-221.

Obata, M., K. Nishimori \& A. Komaru. 2006. Change of centrosome attachment site causes androgenesis in the Freshwater clam Corbicula fluminea: with C. sandai. Venus (Tokyo) 65:247-257.

Palazzo, R. E., J. M. Vogel, B. J. Schnackenberg, D. R. Hull \& X. Wu. 1999. Centrosome maturation. Curr. Top. Dev. Biol. 49:449-470.

Pielak, R. M., V. A. Gaysinskaya \& W. D. Cohen. 2003. Cytoskeletal events preceding polar body formation in activated Spisula eggs. Biol. Bull. 205:192-193.

Pielak, R. M., V. A. Gaysinskaya \& W. D. Cohen. 2004. Formation and function of the polar body contractile ring in Spisula. Dev. Biol. 269:421-432.

Pielak, R. M., C. Hawkins, A. Pyie, J. Bautista, K. G. Lee \& W. D. Cohen. 2005. Polar body formation in Spisula oocytes: function of the peripheral aster. Biol. Bull. 209:21-30.

Powell, E. N., J. Morson \& J. M. Klinck. 2011. Application of a genebased population dynamics model to the optimal egg size problem: why do bivalve planktotrophic eggs vary in size? J. Shellfish Res. 30:403-423.

Schatten, G. 1994. The centrosome and its mode of inheritance: the reduction of the centrosome during gametogenesis and its restoration during fertilization. Dev. Biol. 165:299-335.

Sluder, G., F. J. Miller \& K. Lewis. 1993. Centrosome inheritance in starfish zygotes II: selective suppression of the maternal centrosome during meiosis. Dev. Biol. 155:58-67.

Sluder, G., F. J. Miller \& C. L. Rieder. 1989. Reproductive capacity of sea urchin centrosomes without centrioles. Cell Motil. Cytoskeleton 13:264-273.

Smith, E., N. Hégarat, C. Vesely, I. Roseboom, C. Larch, H. Streicher \& R. Kuriyama. 2011. Differential control of Eg5-dependent centrosome separation by Plk1 and Cdk1. EMBO J. 30:2233-2245.

Steigemann, P. \& D. W. Gerlich. 2009. Cytokinetic abscission: cellular dynamics at the midbody. Trends Cell Biol. 19:606-616.

Stephano, J. L. \& M. C. Gould. 2000. MAP kinase, a universal suppressor of sperm centrosomes during meiosis? Dev. Biol. 222:420-428.

Thiriot-Quiévreux, C., G. H. Pogson \& E. Zouros. 1992. Genetics of growth rate variation in bivalves: aneuploidy and heterozygosity effects in a Crassostrea gigas family. Genome 35:39-45.

Wu, X. \& R. E. Palazzo. 1999. Differential regulation of maternal vs. paternal centrosomes. Proc. Natl. Acad. Sci. USA 96:1397-1402.

Zhang, Q., H. Yu, A. Howe, W. Chandler \& S. K. Allen, Jr. 2010. Cytogenetic mechanism for reversion of triploids to heteroploid mosaics in Crassostrea gigas (Thunberg) and Crassostrea ariakensis. Aquacult. Res. 4:1658-1667.

Zhang, Z., X. Wang, Q. Zhang \& S. K. Allen, Jr. 2014. Cytogenetic mechanism for the aneuploidy and mosaicism found in tetraploid Pacific oyster Crassostrea gigas (Thunberg). J. Ocean Univ. China 13:125-131. 